\title{
Cuando la Compasión Nos Mueve: Manifiesto en el Trabajo
}

\author{
When Compassion Moves Us: It Presents at Work
}

\author{
Luis A. Pedraza González 1", Ramón Rodríguez Montalbán ${ }^{2}$, \& Miguel Martínez Lugo ${ }^{3}$ \\ 1 Albizu University, San Juan, Puerto Rico. (D) https://orcid.org/0000-0002-2910-9144 \\ 2 Albizu University, San Juan, Puerto Rico. (D) https://orcid.org/0000-0003-2414-1943 \\ 3 Albizu University, San Juan, Puerto Rico. (D) https://orcid.org/0000-0001-5586-2327 \\ * Correspondencia: lpedraza@albizu.edu.
}

Recibido: 6 marzo 2021 | Aceptado: 13 agosto 2021 | Publicado: 26 agosto 2021

WWW.REVISTACARIBENADEPSICOLOGIA.COM

\section{Citar como:}

Pedraza González, L.A., Rodríguez Montalbán, R., \& Martínez Lugo, M. (2021). Cuando la compasión nos mueve: Manifiesto en el trabajo. Revista Caribeña de Psicología, 5, e5605. https://doi.org/10.37226/rcp.v5i1.5605

\section{RESUMEN}

La compasión en el trabajo se ha estudiado recientemente como un constructo relevante para promover el bienestar de los empleados, el logro de las metas organizacionales y la calidad de vida organizacional en general. El principal objetivo de este estudio fue analizar las propiedades psicométricas de una batería de ítems con el fin de desarrollar tres escalas para estudiar la compasión en el trabajo (compasión individual, compasión de los compañeros de trabajo y compasión organizacional) en el contexto laboral hispano. La muestra estuvo compuesta por 443 personas de diversos sectores laborales en Puerto Rico. Los resultados confirman una estructura factorial de tres subescalas (cognición, afecto y conductas) para medir la compasión individual, una escala de unidimensional para medir la compasión de los compañeros de trabajo, y una escala con dos dimensiones (políticas y prácticas compasivas), todas buenas propiedades psicométricas. Concluimos que los tres instrumentos para medir la compasión laboral son instrumentos adecuados para su uso en el contexto laboral puertorriqueño, además de ser una aportación a la literatura científica de la psicología ocupacional e industrial organizacional.

Palabras Claves: compasión en el trabajo, psicometría, psicología positiva

\begin{abstract}
Compassion at work has recently been studied as a relevant construct to promote the well-being of employees, the organizational goals' achievement, and the quality of organizational life. The main objective of this study was to analyze the psychometric properties of a battery of items in order to develop three scales to study compassion at work (individual compassion, compassion of co-workers and organizational compassion) in the Hispanic work context. The sample consisted of 443 persons from various labor sectors in Puerto Rico. The results confirm a factorial structure of three sub-scales (cognition, affection and behaviors) to measure individual compassion, a onedimensional scale to measure the compassion of co-workers, and a two-dimensional scale (compassionate policies and practices), all with good psychometric properties. We conclude that the three instruments measuring compassion at work are appropriate instruments for use in the Puerto Rican labor context, in addition to being a contribution to the scientific literature of occupational psychology.
\end{abstract}

Keywords: compassion at work, psychometry, positive psychology 


\section{INTRODUCCIÓN}

La compasión está altamente vinculada a la calidad de vida en el trabajo (Wagaman et al., 2015). A partir de la década del 2000 y hasta el presente, se ha despertado un interés científico por estudiar la compasión en el trabajo desde un paradigma más positivo (Avramchuck \& Manning, 2014; Barbot \& Dodier, 2015; Choi et al., 2016; Chu, 2016; Dutton, Workman \& Hardin, 2014; Gilbert, 2018; Lilius et al., 2008; Ledoux Forchuk et al., 2018). Esta realidad responde a un interés en profundizar acerca de los beneficios individuales y también organizacionales, de la compasión en los contextos laborales. Sin embargo, la revisión de literatura demuestra enorme un vacío del estudio de la compasión laboral en escenarios hispanos y caribeños. La mayoría de las investigaciones de compasión en el trabajo se han realizado en contextos laborales estadounidenses, portugueses, canadienses, ingleses, australianos y asiáticos (Avramchuk \& Manning, 2014; Choi et al., 2016; Dutton et al., 2014; Hur et al., 2014; Ledoux et al., 2018; Lilius et al., 2008; Lupoli et al., 2017; Moon et al., 2014; Ray et al., 2013; Wagaman et al., 2015). Este hallazgo revela la importancia de ampliar la investigación del constructo en los escenarios laborales hispanos y, especialmente, en Puerto Rico.

La estructura formal del trabajo en interacción con otros factores individuales, sociales y ambientales, inciden en el bienestar de los empleados ${ }^{1} \mathrm{y}$ en las metas organizacionales (Lilius et al., 2000). Estos autores señalan que los factores organizacionales pueden provocar incomodidades, malestares, dolores, necesidades y sufrimientos en los empleados. Estas necesidades con mucha frecuencia son observadas por compañeros de trabajo, que, a su vez, buscan hacer algo para aliviar ese sufrimiento, lo que se conoce como conductas compasivas (Lilius et al., 2008). Desde la perspectiva teórica, esta investigación busca retar la conceptualización del sufrimiento como parte inherente a la vida laboral, como se ha planteado hasta el presente en el estudio de la compasión en el trabajo (Barbot et al., 2015; Dutton et al., 2014; Lilius et al., 2008; Worline et al., 2017). Para retar esta conceptualización de la compasión laboral, analizamos la relevancia del

\footnotetext{
${ }^{1}$ Para propósito de este manuscrito, se utilizará el género masculino al referirse a ambos géneros, masculino y femenino, con excepción de cuando se
}

contexto cultural en la definición operacional del constructo, así como su fundamentación teórica.

Entendemos que la compasión en el trabajo no solo es de gran valor para las personas que trabajan en la organización. A nivel organizacional se ha despertado un interés por humanizar los escenarios laborales, hacerlos más sensibles y flexibles (Avramchuk \& Manning, 2014; Lilius et al., 2008). Entonces resulta importante para examinar si las experiencias de compasión son puramente individuales influenciadas por las relaciones interpersonales; o también, son el resultado de prácticas organizacionales que promueven estas conductas.

Un desafío que plantea la literatura revisada es la necesidad de desarrollar y analizar las propiedades psicométricas de escalas que faciliten la medición del constructo desde un espectro más amplio. En la literatura revisada, las escalas existentes solo miden la compasión desde las experiencias de conductas compasivas (Lilius et al., 2008). Sin embargo, para tener una perspectiva amplia de la compasión en el trabajo, es preciso continuar ampliando su estudio desde otras miradas y conceptualizaciones. Particularmente, vemos relevante estudiar la compasión en el trabajo desde escenarios hispanos y diversos, así como entender sus antecedentes y las consecuencias, tanto para el individuo, como para la organización. Para poder entender la compasión en el trabajo, es preciso contar con instrumentos que nos permitan medir adecuadamente el constructo. Para guiar este estudio, nos preguntamos: ¿Cómo podemos se manifiesta la compasión en el trabajo desde la perspectiva individual (cognición, afecto y conductas)? ¿Cómo se manifiesta la compasión desde la perspectiva de otros hacia el individuo (compasión de los compañeros de trabajo)? ¿Cómo se manifiesta la compasión desde la perspectiva organizacional (políticas compasivas y prácticas compasivas)? ¿Cuáles son los procesos psicológicos y sociales que evocan las conductas compasivas?

Este estudio amplía la definición del constructo compasión laboral a partir de la fundamentación teórica y presenta un análisis de los antecedentes y consecuencias de las conductas compasiva en el

esté describiendo un dato específico relacionado al sexo y género de las personas. 
escenario laboral. A su vez, esta investigación reconceptúa el concepto de la compasión en el trabajo y analiza las propiedades psicométricas de tres instrumentos: Escala Individual de Compasión en el Trabajo, Escala de Compasión de Compañeros de Trabajo y la Escala de Compasión Organizacional en una muestra de personas trabajadoras en Puerto Rico. Analizamos las propiedades psicométricas de los tres instrumentos (estructura factorial y confiabilidad), así como la validez convergente y discriminante de nuestras tres escalas de compasión con la Escala de Experiencias de Compasión de Lilius (2008).

\section{La Compasión}

La compasión entre los seres humanos ha sido ampliamente discutida desde distintas vertientes humanistas. Su génesis, principalmente ha estado asociado a contextos espirituales, religiosos, a prácticas de filosofía de vida y a expresiones culturales. En todas estas visiones, se valora y se promueve activamente la empatía y las acciones de compasión hacia otras personas en sufrimiento. Dos aspectos que estas visiones no han podido explicar son: los procesos cognitivos y conductuales involucrados en la compasión como procesos psicológicos y sociales del ser humano y su colectivo.

Para Madden et al. (2012) la compasión es la reacción empática al sufrimiento de otros. Esta reacción está arraigada en un impulso fundamental humano que es la necesidad de ser cuidado, auto-cuidado y en la necesidad de vivir una vida con significado y propósito. Para otros autores (Cameron et al., 2011; Dutton et al., 2014) la compasión es un proceso sistemático influenciado por la cultura, lo que se observa, el significado y lo que se puede hacer para aliviarlo. Estos autores describen cuatro procesos involucrados: 1) identificar el sufrimiento; 2) darle significado al sufrimiento en una forma que contribuya al deseo de aliviar este; 3) sentir preocupación y empatía por las personas que sufren; y 4) tomar acción para aliviar el sufrimiento.

En el contexto laboral, varios autores (Avramchuck et al., 2014; Hur et al., 2016; Rhee, Hur, \& Kim, 2017) conciben la compasión en el trabajo como un proceso de estado mental y también conductual, que involucra empatía consciente hacia el sufrimiento y la angustia de otros que surge como parte de las demandas laborales y el estrés organizacional. Las definiciones de estos autores tienen varios elementos en común: se centran en el sufrimiento observado por otros, las emociones y la empatía que debe resultar en la acción compasiva. Igualmente, coinciden en la importancia del contexto cultural para explicar los procesos involucrados en las conductas compasivas. Sin embargo, estas definiciones no logran explicar los procesos psicológicos involucrados en la manifestación de las conductas compasivas, así como sus antecedentes y consecuencias. Entendemos que estos aspectos son de suma importancia para poder explicar la compasión en los contextos laborales.

En esta investigación definimos compasión laboral como un proceso cognitivo y conductual que involucra estados afectivos estimulados por las necesidades y angustias observadas en otros, que provocan una acción en quien las observa. Estos procesos, que se dan a nivel individual por la interacción social, son influenciados por la organización de forma positiva y también negativa. Para Cameron et al., (2011) estos estados afectivos son el resultado de cuatro procesos interrelacionados: identificación, significado, empatía y acción. En esta investigación estos procesos se reconceptuamos en tres operaciones psicológicas básicas: la operación cognitiva: (a) identificar la necesidad angustiante, y (b) darle significado a la necesidad; lo afectivo: (c) las emociones, (d) sentir preocupación y empatía por la necesidad observada, (e) los recursos que posee el actor compasivo, y (f) su actitud de ayuda; y la operación conductual: (g) la decisión de hacer algo para ayudar de manera que resulte en un alivio o mitigación de la necesidad. Mientras que la compasión de los compañeros de trabajo la definimos como las acciones que realizan otras personas hacia el individuo para mitigar las necesidades angustiantes de la persona.

Por otro lado, la compasión organizacional la definimos como aquellas políticas y prácticas que pone en marcha la organización para mitigar las necesidades angustiantes de los miembros de la organización. Las políticas compasivas son todas aquellas normativas organizacionales (lo que se dice hacer) para mitigar las necesidades angustiantes de los miembros de la organización. Mientras que las prácticas compasivas son las acciones concretas que realiza la organización (lo que se hace), para mitigar las necesidades angustiantes de los miembros de la organización. 


\section{Desarrollo de la Compasión en el Trabajo}

Las primeras investigaciones de compasión influyeron significativamente el estudio del sufrimiento en las organizaciones, lo hicieron desde los aspectos negativos como el malestar y la enfermedad (Davis, 1983). Hasta el presente, hay consenso en los investigadores del tema de compasión laboral al esbozar que el sufrimiento es inevitable e inherente a la vida organizacional (Barbot \& Dodier, 2015; Dutton et al., 2014; Gilbert, 2018; Hur et al., 2016; Lilius et al., 2008; Worline \& Dutton, 2017). Esto en gran medida por las dinámicas, conflictos internos y externos los cuales crean las condiciones para que los empleados manifiesten sufrimiento y angustias. Sin embargo, las investigaciones recientes han dado un giro más positivo al estudio de la compasión (Cameron et al., 2011; Choi et.al., 2016; Chu, 2016; Engen \& Singer, 2015; Hur et al., 2016 Moon et. al., 2016; Wagaman et al., 2015). Aunque reconocen que existe sufrimiento en las organizaciones, se han centrado en los aspectos positivos de las conductas compasivas tales como las emociones positivas, la calidad de vida y el bienestar como resultado de dichas conductas que resultan en la mitigación del sufrimiento (Lilius et al., 2008).

\section{Compasión y Sufrimiento}

El sufrimiento como antecedente de las acciones compasivas ha sido medular en la investigación del constructo hasta el presente. Todas las definiciones de compasión destacan el sufrimiento como parte inherente a la manifestación de las conductas compasivas. Lo que levanta un debate acerca de que, si en efecto, el sufrimiento es el mejor antecedente para explicar la compasión. Eso nos hace cuestionarnos iEs el sufrimiento observado la única manera de activar las acciones compasivas en los contextos laborales y sociales? A nuestro juicio, en el contexto cultural puertorriqueño, el sufrimiento no es necesariamente el antecedente para explicar la compasión laboral. Este debate se fundamenta en la explicación del constructo, desde los contextos culturales y los entendimientos sociales del sufrimiento.

$\mathrm{Al}$ examinar el sufrimiento en el contexto laboral de Puerto Rico, resulta complicado definir el concepto. Por lo general, dentro del lenguaje popular de las personas en Puerto Rico, se adscriben al trabajo formal adjetivos tales como: "el trabajo es honra"; "el trabajo dignifica"; "el trabajo es necesario para subsistir e importante para progresar". Estas frases demuestran, que al menos en Puerto Rico, las personas perciben el trabajo como algo importante en sus vidas (véase Martínez Lugo et al., 1999). Los estudios en Puerto Rico sobre el trabajo han evidenciado que las personas tienden a vincularlo con factores tales como: estrés y ansiedad (Rosario Hernández et al., 2016), incivilidad (Martínez Lugo et al., 2016), emociones negativas (Rosario Hernández et al., 2013), entre otros. Estas investigaciones no reflejan que el trabajo sea percibido como un sufrimiento en los trabajadores en Puerto Rico, más bien, señalan que el trabajo está asociado con otras preocupaciones que generan angustias y pobre calidad de vida laboral.

Según Reich (1989) el sufrimiento es parte de la vida laboral y es el resultado de las experiencias de dolor evocadas esencialmente por angustias. El autor señala que las angustias en el contexto laboral son el resultado de las interacciones entre las situaciones de la vida personal (fuera del trabajo) y la vida laboral (en el trabajo). Para Reich esta definición supone que el concepto sufrimiento involucra emociones, sentimientos, afectos, necesidades (personales y laborales) y angustias. Como resultado de este debate, proponemos que, en el contexto laboral de Puerto Rico, el concepto de necesidades angustiantes resulta ser culturalmente más apropiado para estudiar la compasión laboral y se puso a prueba en esta investigación.

\section{Fundamentación Teórica}

La teoría de los eventos afectivos de Weiss y Cropanzano (1996) establece que las emociones y los estados de ánimo son parte importante de la vida laboral por tanto influyen en el desempeño laboral y en la satisfacción con el trabajo. La teoría de los eventos afectivos explica las manifestaciones de los afectos en los contextos organizacionales, la experiencia personal del trabajo, la percepción personal de su desempeño laboral, y qué sentido tiene este proceso para el trabajador. Esta teoría plantea que las reacciones afectivas son el resultado de las características del entorno en el trabajo, los eventos estresantes y las disposiciones asociadas a la experiencia previa que predisponen a la persona a responder con mayor o menor intensidad a los eventos angustiantes. Esto explica la fase de operación cognitiva en la compasión (observación de un evento de expresiones de angustia y darle significado). Por otro lado, los procesos psicológicos 
y cognitivos tales como las emociones, las interpretaciones y los significados de los eventos estresantes, unido a las experiencias previas, desencadenan reacciones afectivas (Velasco et al., 2015). Esto puede explicar la fase de operación afectiva en la compasión (las emociones y actitudes). Finalmente, los procesos conductuales del modelo incluyen el comportamiento, las actitudes en el trabajo, las conductas dirigidas a los afectos y al juicio. Esto explica la fase de operación conductual (la conducta compasiva). Para este modelo los recuerdos afectivos positivos, una vez ocurren, promoverían futuras acciones compasivas.

La teoría de apego de Bowlby $(1969 ; 1973)$ señala que las reacciones de las personas a las necesidades de los demás, surgen como consecuencia de un sistema de cuidado generado por el apego y la confianza. Las personas que sienten apego hacia sus compañeros de trabajo se sienten seguros y tienen la expectativa de que estos serán recurso de apoyo cuando tengan alguna necesidad angustiante. En el proceso de la compasión en el trabajo, estos componentes explican la operación afectiva (la empatía y los afectos) y la operación conductual (actitud y la acción compasiva).

La teoría del intercambio social de Blau (1964) explica la interacción entre el actor compasivo y la persona en necesidad. Blau postula que esa interacción parte de procesos psicológicos y del intercambio de recompensas para ambas partes. Las personas se asocian por simpatía y afinidad y ello da inicio a una serie de intercambios para obtener beneficios y en el caso de la compasión obtener el bienestar del otro. Esta teoría explica que las interacciones sociales entre los individuos van desde las asociaciones elementales básicas hasta otras más complejas como el intercambio de grandes asociaciones que a su vez imita el colectivo.

\section{Antecedentes y Consecuencias de la Compasión en el Trabajo}

Varios autores destacan que los antecedentes de las conductas compasivas surgen a partir de la asociación entre procesos cognitivos y afectivos (Avramchuck et al., 2014; Cameron et al., 2011; Hur et al., 2016; Rhee et al., 2017). El primero de estos procesos son las operaciones cognitivas, las cuales involucran algún evento angustiante en la persona, seguido por la expresión de necesidad, y finalmente el significado a las emociones observadas. El segundo proceso son las operaciones afectivas, las cuales involucran las emociones, la empatía de la persona que observa la necesidad del otro, los recursos personales del observador y la actitud de ayudar. De acuerdo a estos procesos, una de las primeras conductas observadas en situaciones angustiantes son los cambios de estados de ánimo. Estos cambios de ánimo observado en otras personas provocan que se activen las operaciones cognitivas y afectivas.

Lilius et al. (2008) destacan que el sufrimiento observado en el otro, las angustias y las necesidades son el detonante y antecedente principal para provocar conductas compasivas. A su vez, la relación entre las dinámicas del contexto laboral tóxico, el ambiente y su interacción con las realidades individuales, tanto personal como laboral, inciden en la manifestación de conductas compasivas (Barbot \& Dodier, 2015; Dutton et al., 2014; Lilius et al., 2008; Gilbert, 2018; Reich, 1989; Worline et al., 2017). Ello despierta un interés de las personas por ayudar y aliviar el sufrimiento lo cual tiene un rol de antecedente de la compasión laboral (Cameron et al., 2011).

Cabe destacar que hay aspectos positivos que también pueden ser desencadenantes de la compasión laboral. En su estudio, Chu (2016) encontró que los estados de ánimo positivos pueden ser antecedentes de la compasión en el trabajo. En la medida que las personas manifiestan estados de ánimo positivos, demuestran mayor sensibilidad hacia las necesidades de otras personas y disposición a actuar para aliviar al otro. Las percepciones de los empleados sobre la responsabilidad social corporativa, la motivación intrínseca (Hur et al., 2016ª ; Moon et al., 2014), la justicia organizacional y el compromiso afectivo con el trabajo (Moon et al., 2014, 2016), también han sido considerados como antecedentes de las conductas compasivas.

En cuanto a las consecuencias de la compasión en el trabajo, múltiples investigaciones evidencian que la compasión en el trabajo está positivamente asociada con el funcionamiento de los empleados y de la organización en general (Cameron, 2017; Cameron et al., 2011; Choi et al., 2016; Dutton et al., 2007; Hur et al., 2016b; Lilius et al., 2008; Madden et al., 2012). Cabe destacar que las dinámicas organizacionales provocan que los seres humanos que laboran en ellas 
desarrollen sus propios mecanismos para atender sus necesidades de un modo orgánico. De esta manera, la compasión es descrita como una experiencia positiva que puede manifestarse con mucha naturalidad en una organización (Cameron et al., 2011; Choi et al., 2016). En ese sentido, las manifestaciones de compasión en el trabajo tienen como consecuencias experiencias positivas que fortalecen la calidad de vida laboral y crean las condiciones para que se repitan (Cameron et al., 2011). De esta forma, se desarrollan mecanismos sistémicos informales que tienen el propósito de amortiguar el efecto de las necesidades angustiantes en el trabajo (Bowlby, 1973).

Lilius et al. (2008) encontraron que las experiencias de compasión en el trabajo son importantes para experimentar emociones positivas y compromiso afectivo, y en consecuencia este efecto perdura a través del tiempo y es acumulativo. Además, los autores encontraron que las emociones positivas como antecedentes median la relación entre las experiencias de compasión y el compromiso afectivo. Por su parte, Choi et al. (2016) encontraron que la compasión en el trabajo alivia las emociones negativas como la ansiedad, el burnout y la rotación. También se ha encontrado que la compasión en el trabajo reduce la intención de abandono (Choi et al., 2016; Moon et al., 2016), aumenta el desempeño (individual y organizacional) y los comportamientos de ciudadanía organizacional (Chu, 2016; Hur et al., 2016), así como la creatividad de los empleados (Hur et al., 2016), la identidad positiva y el compromiso afectivo (Moon et al., 2016), el apoderamiento psicológico y la colaboración entre profesionales (Ledoux et al., 2018)

\section{Medición de la Compasión Laboral}

La escala más utilizada para la medición de las experiencias de compasión laboral fue desarrollada por Lilius et al., (2008) en los Estados Unidos y es denominada Escala de Experiencias de Compasión en el Trabajo. La escala cuenta con tres ítems que miden la frecuencia de las experiencias de compasión en el trabajo a nivel personal, por su supervisor y por parte de compañeros. A pesar de que este instrumento es el más utilizado en el estudio de la compasión, tiene una seria limitación y es que solo mide la frecuencia de las conductas compasivas en el contexto laboral.

Otra escala utilizada para medir este constructo es la Escala de Compasión de Pommier (2010) desarrollada en Portugal y consta de seis subescalas para medir: amabilidad, humanidad, atención plena, indiferencia, separación y retirada. Esta escala también mide la frecuencia de las experiencias de compasión desde la perspectiva individual. Una tercera escala para medir compasión está incluida en la Escala Profesional de Calidad de Vida (Professional Quality of Life Scale, Pro$Q O L)$ desarrollada por Stamm (2010) en Australia. Esta consta de tres subescalas para medir: satisfacción por compasión, burnout, el estrés traumático secundario. La subescala de satisfacción por compasión también mide la experiencia individual de compasión laboral.

Pese a sus aportaciones al entendimiento de la compasión laboral, estos instrumentos obvian hasta cierto punto los componentes cognitivos y afectivos del constructo. A su vez, solo se centran en las conductas compasivas de otros hacia la persona que responde el instrumento, pues no toma en cuenta la compasión del individuo hacia otros, ni tampoco los componentes organizacionales (políticas y prácticas) que pueden promover la compasión. Para atender estas limitaciones, elaboramos tres instrumentos que pretenden medir la compasión laboral desde tres perspectivas: la compasión del individuo hacia otros, la compasión de otros hacia el individuo y los factores organizacionales que promueven la compasión. Cabe destacar que en la literatura científica revisada se evidencia un enorme vacío del estudio de la compasión laboral en el contexto hispano.

\section{Propósito del Estudio}

Tal como señalamos antes, esta investigación tiene dos propósitos: 1) reconceptuar el fenómeno de la compasión laboral y 2) analizar las propiedades psicométricas de tres instrumentos para medir la compasión en el entorno laboral. Aunque el estudio de la compasión en el trabajo ha aumentando en los últimos años, hasta el momento no encontramos estudios del fenómeno en los contextos latinoamericanos incluyendo a Puerto Rico. Hasta el presente, la medición de la compasión laboral se ha ceñido desde las experiencias individuales en una sola dirección (Lilius et al., 2008; Pommier, 2010; Stamm, 2010), más no su manifestación bidireccional, ni de tipo estructural organizacional. Por tanto, en este estudio analizamos las propiedades psicométricas de tres instrumentos (Escala Individual de Compasión en el Trabajo, Escala de 
Compasión de Compañeros de Trabajo y Escala de Compasión Organizacional), con el propósito de tener una perspectiva más amplia del fenómeno de la compasión laboral, desde diferentes aspectos dentro del entorno organizacional.

\section{MÉTODO}

\section{Diseño de Investigación}

La investigación se llevó a cabo mediante un diseño no experimental, transversal, de tipo instrumental (Ato et al., 2013; Montero \& León, 2007), empleando análisis de factores confirmatorios con ecuaciones estructurales. Las investigaciones no experimentales observan el fenómeno tal y como se da en su contexto natural (Hernández Sampieri et al., 2006). Este estudio tuvo como objetivo hacer alusión a que primero se produce el hecho y después se analizan las posibles causas y consecuencias, por lo que se trata de un tipo de investigación en donde no se modifica el fenómeno o situación objeto de análisis (Mertens, 2004).

\section{Participantes}

La muestra fue obtenida por disponibilidad y estuvo compuesta por 526 participantes que completaron el cuestionario de compasión en el trabajo. Luego de un proceso de limpieza de los datos y eliminación de cuestionarios incompletos, se incluyeron en la muestra un total de 443 participantes, lo que representa una taza de respuesta de $84.2 \%$. Un $54.2 \%$ indicó pertenecer al género femenino, el $44.7 \%$ al género masculino, y un $1 \%$ se identificó como transgénero. Las edades de los participantes oscilaron entre 21 a 78 años $(M=36.67, D E=11.73)$. La mayoría de los participantes indicaron: poseer un grado de maestría $(44 \%)$, trabajar a tiempo completo $(65.2 \%)$, trabajar en la empresa privada (78.3\%), laborar dentro del sector de servicios (33.9\%) y no realizar tareas de supervisión $(73.1 \%)$. El número de años trabajando de los participantes fluctuó entre menos de un año a 49 años $(M=10.08, D E=10.02)$.

\section{Instrumentos}

Hoja de datos demográficos. En este instrumento se solicitó información referente al género de los participantes, edad, preparación académica, estado civil, número de años trabajando, tipo de organización donde trabaja, tipo de industria para la cual trabaja, número de horas trabajadas a la semana, si realiza tareas de supervisión y tipo de contrato que tiene con la organización.

Escala de Experiencias de Compasión en el Trabajo. La escala fue elaborada por Lilius et al., (2008), es de auto reporte y consta de tres ítems que miden la frecuencia de las experiencias de compasión en el trabajo a nivel individual. Su formato de respuesta es de tipo Likert de 5 puntos que fluctúa entre $1=$ nunca y 5 = todo el tiempo. Un ejemplo de un ítem es "¿Con qué frecuencia experimenta compasión en el trabajo?". En este estudio la escala tuvo un índice de fiabilidad alfa de Cronbach de $\alpha=.89$.

Cuestionario de Compasión en el Trabajo. Este cuestionario tuvo 55 ítems desarrollados por Pedraza, Rodríguez Montalbán y Martínez divididos en tres perspectivas: (a) las expresiones de compasión hacia otros (ítems 1-24), (b) las expresiones de compasión de otros hacia mí (ítems 25-35), (c) lo que la organización hace para estimular conductas compasivas (ítems 36-55). El instrumento tiene un anclaje de respuesta que va de 0 (Nunca) hasta 4 (Siempre). Un ejemplo de un ítem de la dimensión de expresiones de compasión hacia otros es: "Cuando un compañero tiene una necesidad angustiante, busco la manera de ayudarlo"; un ejemplo de un ítem de la dimensión de las expresiones de compasión de otros hacia mí es: "Cuando me siento triste o agobiado, mis compañeros hacen algo para ayudarme"; y un ejemplo de un ítem de la dimensión sobre lo que la organización hace para estimular conductas compasivas es: "La organización donde trabajo estimula que se generen conductas compasivas entre los empleados".

\section{Procedimientos Generales}

En este estudio se utilizaron datos secundarios de la investigación realizada por Rodríguez-Montalbán (2018) para lo cual se obtuvo autorización del autor. El estudió contó con la aprobación del comité de revisión institucional de la Universidad Carlos Albizu de San Juan, Puerto Rico (protocolo Fall-19-24). Se invitó a las personas a participar del estudio completando los cuestionarios en línea y en lápiz y papel. Se garantizó la participación voluntaria, el anonimato y el derecho a abandonar el estudio cuando lo consideraran necesario sin ser penalizados. Luego de obtener el permiso de uso de los datos recopilados por parte del investigador, se procedió a conformar la base de datos de esta investigación para su posterior análisis. 


\section{Análisis de Datos}

Los análisis estadísticos realizados fueron: análisis descriptivos (medias, desviaciones, asimetría y curtosis), análisis de normalidad univariante (Kolmogorov Smirnov y Shapiro Wilk), análisis de normalidad multivariante ( $M$ de Mardia, Doornik-Hansen y Henze-Zirkler con el programa STATA versión 15), análisis de factores confirmatorio con el método de estimación de máxima verosimilitud (ecuaciones estructurales con el programa AMOS versión 25) y las correcciones de los índices de bondad de ajuste de Satorra y Bentler (2001) para datos no normales (con el programa $R$ ), análisis de reactivos (correlación ítem total), estimación de la fiabilidad (alfa de Cronbach y fiabilidad compuesta) y el análisis de correlación $r$ de Pearson de todas las escalas. Por último, se procedió a examinar la varianza media extraída de las escalas como recomiendan Fornell y Lacker (1981). Se analizó la validez discriminante de la Escala de Experiencias de Compasión en el Trabajo (Lilius et al., 2008) con la Escala Individual de Compasión en el Trabajo, la Escala de Compasión de Compañeros de Trabajo y la Escala de Compasión Organizacional.

\section{RESULTADOS}

\section{Análisis Descriptivos de los Ítems}

En esta investigación se calcularon las medias y desviaciones estándar para los 24 ítems del cuestionario de compasión en el trabajo. Las medias fluctuaron entre 1.92 y 3.32, con desviaciones estándar que fluctuaron entre .82 y 1.32. Los estadísticos de normalidad de la muestra según Kolmogorov-Smirnov y Shapiro-Wilk validaron que los datos no siguen una distribución normal. Los resultados del análisis de normalidad multivariante confirman que no hay normalidad multivariante de los datos: $M$ de asimetría = 821.11, $X^{2}$ (32509) 61050.09, $p<.001, M$ de curtosis $=$ 4093.57, $X^{2}(1)$ 8788.61, $p<.001$, Henze-Zirkler $=1.03, X^{2}$ (1) $.00, p<.001$, Doornik-Hansen $=X^{2}$ (114) 1487.86, $p$ $<.001$. Dado a que no se cumple el supuesto de normalidad multivariante, se utilizaron las correcciones de Satorra y Bentler (2001) para los índices de bondad de ajuste de los modelos de ecuaciones estructurales.

\section{Análisis Factorial de las Escalas de Compasión}

En esta investigación se pusieron a prueba un total de 55 ítems del cuestionario de compasión en el trabajo para examinar su estructura factorial. Para ello, se realizó un análisis confirmatorio de factores con ecuaciones estructurales mediante el método de estimación de máxima verosimilitud. Primero pusimos a prueba un modelo unifactorial con todos los ítems de compasión. Este modelo no presentó buenos índices de ajuste a los datos: $X^{2}=4254.224(1430) p<.001$, RMSEA $=.12, C F I=.63, N F I=.59$, IFI $=.63$, AIC $=$ 11211.29, $X^{2}$ corregido $=1099.29(1430) p<.001, C F I$ corregido $=.49$, NFI corregido $=.46$, IFI corregido $=$ .49 , AIC corregido $=11064.85$. Estos resultados son indicativos de que los ítems se deben modelizar en más de un factor latente. Luego, analizamos la estructura factorial en dos niveles (individual y organizacional). Realizamos un proceso interactivo de análisis y se fueron eliminando ítems con cargas factoriales menores a .65 como según recomendado (Brown, 2015). Como resultado de este análisis fueron eliminados un total de 31 ítems que no presentaron cargas factoriales superiores a .65. El primer nivel factorial agrupó las dos escalas de compasión a nivel individual: Escala de Compasión Individual (la compasión de mí hacia otros) y la Escala de Compasión de Compañeros de Trabajo (la compasión de otros hacia mî). Primero analizamos la Escala de Compasión Individual (dimensiones de cognición, afecto y conductas compasivas). La Figura 1 evidencia que este modelo tuvo un buen ajuste para explicar la compasión desde estos tres subdominios. El análisis confirmatorio de factores demostró que este modelo posee buen ajuste a los datos según Satorra y Bentler (2001): $X^{2}=104.005$ (24) $p<$ .001, RMSEA $=.08, C F I=.97, N F I=.96, I F I=.97, A I C=$ $146.01, X^{2}$ corregido $=60.182$ (24) $p<.001$, CFI corregido $=.97$, NFI corregido $=.96$, IFI corregido $=.97$, AIC corregido $=106.20$.

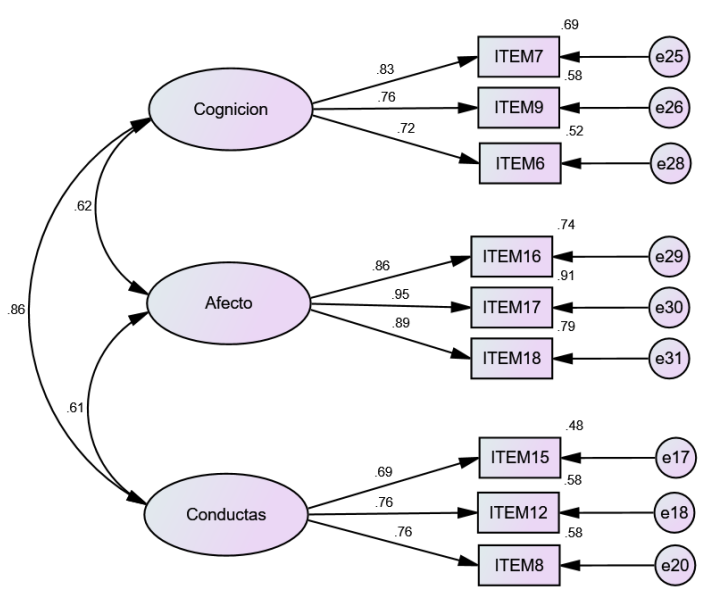

Figura 1. Escala Individual de Compasión en el Trabajo 
Luego analizamos la Escala de Compasión de Compañeros de Trabajo (la compasión de otros hacia mí) compuesta por una dimensión de siete ítems (ver Figura 2). Este modelo también presentó un buen ajuste factorial: $X^{2}=60.643(14) p<.001, R M S E A=.08$, $C F I=.97, N F I=.97, I F I=.97, A I C=88.643, X^{2}$ corregido $=46.182(14) p<.001$, CFI corregido $=.98$, NFI corregido $=.97$, IFI corregido $=.98$, AIC corregido $=61.62$.

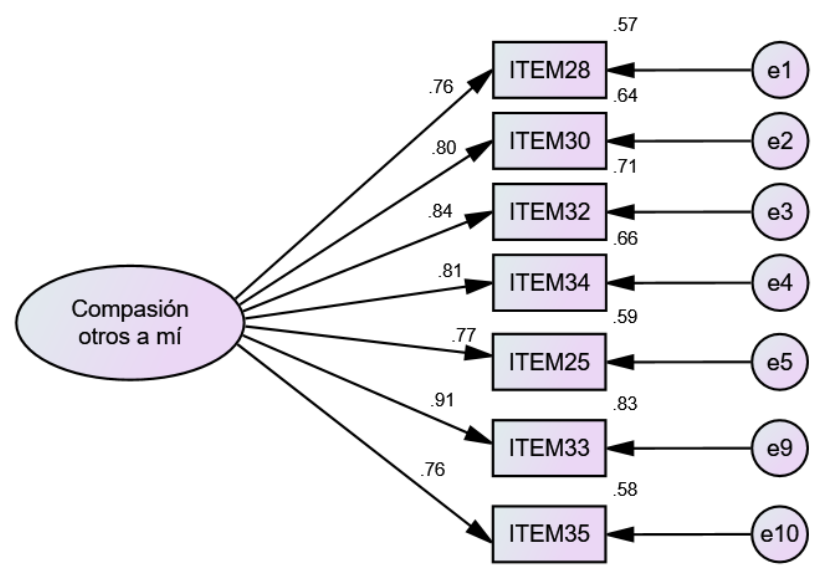

Figura 2. Escala de Compasión de Compañeros de Trabajo

Por último, analizamos la Escala de Compasión Organizacional compuesta por ocho ítems, donde los agrupamos en dos dimensiones: tres ítems para las políticas organizacionales compasivas y cinco ítems para las prácticas organizacionales compasivas. Este modelo también presentó un buen ajuste a los datos: $X^{2}=60.282$ (19) $p<.001$, RMSEA $=.07, C F I=.97, N F I=.97, I F I=.98$, $A I C=94.282, X^{2}$ corregido $=50.162$ (19) $p<.001, C F I$ corregido $=.98$, NFI corregido $=.97$, IFI corregido $=$ .98, AIC corregido $=61.72$ (ver Figura 3 ).

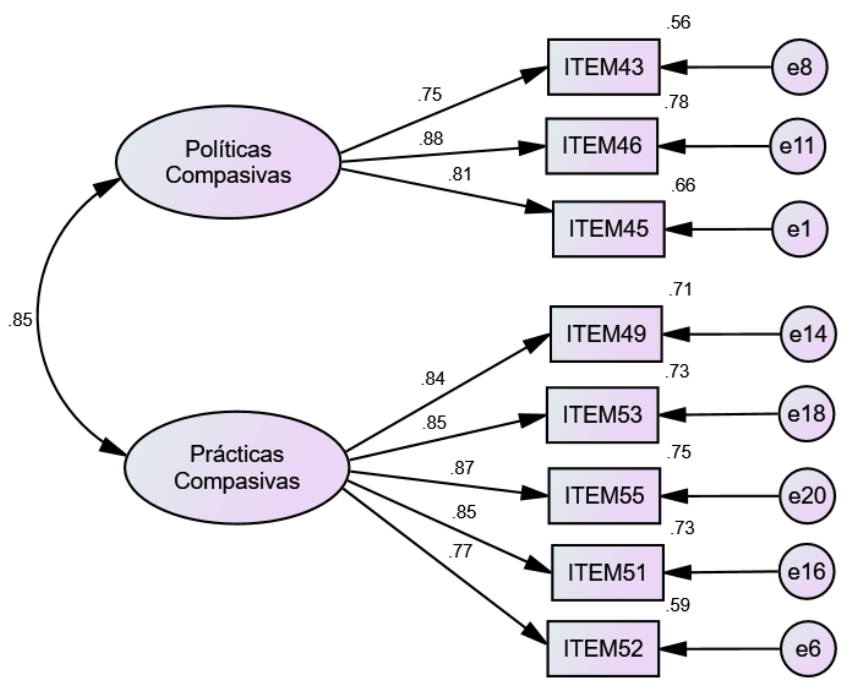

Figura 3. Escala de Compasión Organizacional

\section{Análisis de los Ítems}

Se examinó la capacidad de discriminación de los ítems compasión en el trabajo para cada una de las escalas, mediante el análisis de los índices de correlación ítem total. En la Escala de Compasión Individual (de mí hacia otros) los índices de discriminación oscilan entre .69 y .95 , mientras que la varianza explicada en los ítems oscila entre 48 y .91. Para la Escala de Compasión de Compañeros de Trabajo (de otros hacia mî) los índices de discriminación oscilan entre .76 y .91, mientras que la varianza explicada en los ítems oscila entre .57 y .71. Para la Escala de Compasión Organizacional (políticas organizacionales compasivas y prácticas organizacionales compasivas) los índices de discriminación oscilan entre .75 y .88. Mientras que la varianza explicada en los ítems oscila entre .56 y .78 (véase Tablas 1, 2 y 3). Todos los índices de discriminación de las tres subescalas están por encima del mínimo recomendado de .30 (Kline, 2005)

Tabla 1

Índices de discriminación y varianza explicada de la Escala Individual de Compasión

\begin{tabular}{|c|c|c|}
\hline Ítems & $\begin{array}{c}\text { Índice de } \\
\text { discriminación }\end{array}$ & $H^{2}$ \\
\hline $\begin{array}{l}\text { 1. Cuando observo a un compañero } \\
\text { muy estresado busco la manera de } \\
\text { aliviar su estrés. }\end{array}$ & .72 & .52 \\
\hline $\begin{array}{l}\text { 2. Estoy pendiente de las necesidades } \\
\text { de mis compañeros. }\end{array}$ & .83 & .69 \\
\hline $\begin{array}{l}\text { 3. Estoy pendiente de las necesidades } \\
\text { angustiantes de mis compañeros ya } \\
\text { sea por asuntos de trabajo o por asun- } \\
\text { tos personales. }\end{array}$ & .76 & .58 \\
\hline $\begin{array}{l}\text { 4. Me siento intranquilo cuando mis } \\
\text { compañeros están sufriendo. }\end{array}$ & .86 & .74 \\
\hline $\begin{array}{l}\text { 5. Me siento angustiado cuando mis } \\
\text { compañeros están sufriendo. }\end{array}$ & .95 & .91 \\
\hline $\begin{array}{l}\text { 6. Me siento triste cuando mis compa- } \\
\text { ñeros están sufriendo. }\end{array}$ & .89 & .79 \\
\hline $\begin{array}{l}\text { 7. Le pregunto a mis compañeros si } \\
\text { están bien y si necesitan ayuda. }\end{array}$ & .76 & .58 \\
\hline $\begin{array}{l}\text { 8. Busco la manera de ayudar a mis } \\
\text { compañeros de trabajo cuando lo ne- } \\
\text { cesitan. }\end{array}$ & .76 & .58 \\
\hline $\begin{array}{l}\text { 9. Es mi naturaleza ayudar a la gente } \\
\text { cuando lo necesitan. }\end{array}$ & .69 & .48 \\
\hline
\end{tabular}

Nota: Ítems 1 - 3 = Cognición; $4-6$ = Afecto; Ítems 7 -9= Conducta; $H^{2}=$ comunalidad. 
Tabla 2

Índices de discriminación y varianza explicada de la Escala Compasión de Compañeros de Trabajo

\begin{tabular}{lcc}
\hline \multicolumn{1}{c}{ Ítems } & rbis & $H^{2}$ \\
\hline $\begin{array}{l}\text { 1. Cuando me siento triste o agobiado, mis compañe- } \\
\text { ros hacen algo para ayudarme. }\end{array}$ & .59 & .59 \\
2. Recibo muestras de apoyo (por ejemplo: palabras de & .76 & .57
\end{tabular}

aliento o abrazos) de mis compañeros cuando notan que estoy agobiado en el trabajo.

3. Mis compañeros demuestran empatía hacia mí $.80 \quad .64$ cuando tengo una necesidad angustiante.

4. Mis compañeros se ponen en mi lugar cuando notan que estoy angustiado por el trabajo o por asuntos personales.

5. Mis compañeros me consuelan cuando notan que estoy angustiado por el trabajo o por asuntos personales.

6. Mis compañeros se muestran cariñosos conmigo cuando notan que estoy angustiado por el trabajo o por asuntos personales.

7. Mis compañeros dicen presente cuando necesito ha- $\quad \begin{array}{lll}.76 & .58\end{array}$ blar con alguien de mis problemas.

Nota: $H^{2}=$ comunalidad; rbis = Índice de discriminación.

Tabla 3

Índices de discriminación y varianza explicada de la Escala Compasión Organizacional

\begin{tabular}{lccc}
\hline \multicolumn{1}{c}{ Ítems } & rbis & $R^{2}$ \\
\hline $\begin{array}{l}\text { 1. En mi trabajo existen políticas sensibles a las necesi- } \\
\text { dades o angustias de los empleados/as. }\end{array}$ & .75 & .56 \\
$\begin{array}{l}\text { 2. En mi trabajo, los supervisores se ponen en el lugar } \\
\text { de los empleados cuando estos tienen situaciones per- }\end{array}$ & .81 & .66 \\
$\begin{array}{l}\text { sonales que afectan el trabajo. } \\
\text { 3. En mi trabajo se valoran las conductas compasivas }\end{array}$ & .88 & .78 \\
entre empleados/as. & & \\
$\begin{array}{l}\text { 4. La organización donde trabajo estimula que se gene- } \\
\text { ren conductas compasivas entre los empleados. }\end{array}$ & .84 & .71 \\
$\begin{array}{l}\text { 5. La organización donde trabajo hace cosas para ali- } \\
\text { viar la situación de los empleados/as cuando están su- }\end{array}$ & .85 & .73 \\
friendo. & & \\
$\begin{array}{l}\text { 6. La organización altera sus políticas institucionales } \\
\text { para implantar acciones que benefician a empleados/as }\end{array}$ & .77 & .59 \\
$\begin{array}{l}\text { que están atravesando una situación angustiante. } \\
\text { 7. La organización promueve que se ayude a los com- }\end{array}$ & & & \\
pañeros cuando están atravesando una situación difí- & & & \\
cil. & & & \\
$\begin{array}{l}\text { 8. La organización promueve las conductas compasi- } \\
\text { vas entre empleados/as. }\end{array}$ & .87 & .75 \\
\hline
\end{tabular}

Nota: Ítems 1 - 3 = Políticas Compasivas; Ítems 4 - 8 = Prácticas Organizacionales Compasivas; rbis = Índice de discriminación.

\section{Análisis de Confiabilidad de las Escalas de Compa- sión Laboral}

Como parte de los objetivos de esta investigación, se analizó la confiabilidad de la versión final del cuestionario de compasión en el trabajo. Los valores alfa de Cronbach fluctuaron entre .78 y .93, mientras que los valores omega de McDonald oscilan entre .78 y 93. En la Tabla 4 se presentan los valores alfa de Cronbach y omega de McDonald para cada factor latente. Se analizó además la confiabilidad y fiabilidad de la escala de experiencias de compasión de Lilius et al. (2008), se obtuvo un alfa de Cronbach de .83 y un índice omega de McDonald de .84 .

\section{Análisis de Validez Convergente y Discriminante de las Escalas de Compasión Laboral}

Se analizó la validez convergente y discriminante mediante el uso de la técnica de comparación de la Varianza Media Extraída (VME). La VME mide el nivel de varianza explicada por el factor latente en los ítems. Los valores altos en la VME indican que la varianza de error es menor. Los valores VME de todos los constructos del cuestionario de compasión en el trabajo fluctuaron entre .55 y .81 (véase Tabla 4), por lo que según Fornell y Larcker (1981) son valores aceptables al ser igual o mayor a .50. Además, se realizó un análisis de la correlación entre las dimensiones del cuestionario mediante la correlación de Pearson. La Tabla 4 presenta las correlaciones de todas las escalas con sus dimensiones. Las correlaciones $r$ de Pearson entre las dimensiones fluctuaron entre .27 y .76. La varianza compartida entre dos constructos siempre es menor a la varianza explicada por cada uno de ellos, lo cual cumple con el criterio de validez discriminante propuesto por Fornell y Larcker (1981). Además, los resultados evidencian que cada una de las dimensiones de cada subescala, mide un constructo particular sin que haya redundancia entre los factores.

Por otro lado, se analizó la validez convergente de las escalas de compasión en el trabajo con la escala de experiencias de compasión de Lilius (2008). Para esto, se realizó el análisis de correlación de Pearson de la escala de Lilius con cada una de las escalas de compasión en el trabajo y sus dimensiones. Los índices de correlación fluctuaron entre .34 y .67. La varianza compartida entre las escalas de compasión y la escala de Lilius fluctuaron entre .12 y .45. La Varianza Media 
Extraída de la escala de Lilius (2008) alcanzó un índice de .64. Se concluye que todas las escalas miden diferentes manifestaciones de la compasión laboral

\section{DISCUSIÓN}

El propósito de esta investigación fue elaborar una definición del constructo de la compasión en el trabajo (individual, de compañeros de trabajo y organizacional), a partir de la fundamentación teórica, así como el elaborar tres instrumentos de compasión laboral a partir de nuestra operacionalización del constructo. Los resultados del análisis psicométrico de las escalas de compasión laboral revelan: 1) que la compasión del individuo hacia otros posee tres componentes (cognitivo, afectivo y conductual); 2) que las manifestaciones de las conductas compasivas de otros hacia que buscan aliviar las necesidades angustiantes de la persona son unidimensionales; 3) que la compasión a nivel organizacional se puede concebir desde dos componentes (las políticas compasivas y las prácticas compasivas).

\section{Implicaciones Teóricas y Prácticas}

A nivel teórico, nuestros hallazgos validan y resaltan la importancia de ampliar la definición de la compasión en el entorno laboral, para que permita distinguir los tipos de compasión, así como los niveles del constructo (individual y organizacional). Nuestro estudio logra ampliar, profundizar y explicar con mayor especificidad los procesos psicológicos involucrados en las conductas compasivas en el trabajo, a partir de teorías ampliamente estudiadas (teoría de los eventos afectivos, teoría de apego y teoría de intercambio social), robustas y de fácil comprensión. Esto nos permite un mejor entendimiento de los procesos psicológicos y sociales involucrados en las manifestaciones de conductas compasivas en el trabajo. Además, nuestra noción de la compasión en el trabajo amplía las definiciones de la literatura científica, al no solo explicar estos procesos, sino que también explica los antecedentes y las consecuencias de estas conductas.

A nivel individual (del individuo hacia otros), evidenciamos tres operaciones psicológicas básicas: cognitiva, afectiva y conductual. Esta nueva definición estuvo fundamentada en una robusta fundamentación teórica: 1) la teoría de eventos afectivos de Weiss y Cropanzano (1996) para explicar los procesos cognitivos, para interpretar las necesidades angustiantes y adscribirle significado; 2) la teoría de apego de Bowlby $(1969 ; 1973)$ para explicar la conducta compasiva a la luz de un sistema de cuidado producido por el apego y el sentido de seguridad; y 3) la teoría de intercambio social de Blau (1964) para explicar el intercambio de las conductas compasivas que son el resultado de las interacciones sociales para el beneficio de otros, de sí mismo y que tienen el efecto de repetirse e imitarse.

Del mismo modo, nuestra noción de la compasión de los compañeros de trabajo se distancia de la noción tradicional del sufrimiento, para darle otro matiz,

\section{Tabla 4}

Medias, desviaciones estándar, alfas, fiabilidad compuesta, varianza media extraída y correlaciones $(N=443)$

\begin{tabular}{|c|c|c|c|c|c|c|c|c|c|c|c|}
\hline & $M$ & $D E$ & $\alpha$ & $\Omega$ & $V M E$ & 1 & 2 & 3 & 4 & 5 & 6 \\
\hline 1. Cognición & 2.68 & .84 & .81 & .82 & .60 & - & - & - & - & - & - \\
\hline 2. Afecto & 2.54 & 1.04 & .93 & .93 & .81 & $.56^{* *}$ & - & - & - & - & - \\
\hline 3. Conductas & 3.22 & .71 & .78 & .78 & .55 & $.68 * *$ & $.54 * *$ & - & - & - & - \\
\hline $\begin{array}{l}\text { 4. Compasión de } \\
\text { otros hacia mí }\end{array}$ & 2.48 & .88 & .93 & .93 & .65 & $.52 * *$ & $.46^{* *}$ & $.42 * *$ & - & - & - \\
\hline $\begin{array}{l}\text { 5.Políticas } \\
\text { compasivas }\end{array}$ & 2.40 & 1.08 & .85 & .86 & .66 & $.29 * *$ & $.27 * *$ & $.29 * *$ & $.54 * *$ & - & - \\
\hline $\begin{array}{l}\text { 6. Prácticas } \\
\text { organizacionales }\end{array}$ & 2.24 & 1.07 & .90 & .92 & .70 & $.33 * *$ & $.29 * *$ & $.30 * *$ & $.49 * *$ & $.76^{* *}$ & - \\
\hline $\begin{array}{l}\text { 7. Escala de } \\
\text { Experiencias de } \\
\text { Compasión (Lilius, } \\
\text { 2008) }\end{array}$ & 3.37 & .90 & .83 & .84 & .64 & $.39 * *$ & $.34 * *$ & $.35^{* *}$ & $.58 * *$ & $.67 * *$ & $.63 * *$ \\
\hline
\end{tabular}

Nota: $M=$ media; $D E=$ desviación estándar; $\alpha=$ alfa de Cronbach; $\Omega=$ omega de McDonald; $V M E=$ varianza media extraída; $* *=$ correlaciones significativas a $p<.001$ 
partiendo de las necesidades angustiantes que puede presentar un individuo. En este sentido, vemos la compasión de los compañeros de trabajo como las acciones que realizan otras personas para mitigar estas necesidades angustiantes y fomentar un contexto laboral de apoyo y colaboración. La investigación ha evidenciado que este tipo de conductas promueven el desempeño (Chu, 2016), el compromiso afectivo (Moon et al., 2016) y la colaboración entre los compañeros de trabajo (Ledoux et al., 2018).

A nivel organizacional, nuestra noción de la compasión a nivel macro, posee dos características distintivas: 1) las políticas organizacionales que buscan promover la compasión y 2) las prácticas organizacionales que buscan mitigar las necesidades angustiantes de los miembros de la organización. Esta noción de la compasión permitiría tener un mejor entendimiento de aquellas prácticas que pueden poner en marcha las organizaciones para promover ambientes de trabajo que sean sensibles a las necesidades de los miembros de la organización, así como el promover el bienestar dentro del contexto organizacional.

A partir de estas nociones de la compasión en el trabajo, nuestro estudio cumplió con el objetivo de desarrollar y analizar las propiedades psicométricas de tres instrumentos (la Escala Individual de Compasión en el Trabajo, la Escala de Compasión de Compañeros de Trabajo y la Escala de Compasión Organizacional) para medir la compasión en el escenario laboral en el contexto hispano. De los resultados obtenidos, podemos concluir que nuestros instrumentos son apropiados para estudiar la compasión laboral desde tres perspectivas (la compasión del individuo hacia otros, la compasión de otros hacia el individuo y la compasión a nivel organizacional). Estas tres perspectivas amplían la forma de entender y estudiar la compasión en el trabajo, ya que toma en consideración los procesos individuales y también organizacionales, distinto a como lo hacen otras escalas (Lilius et al., 2008; Pommier, 2010; Stamm, 2010), las que solo toman en consideración los aspectos individuales y experienciales de la compasión. Nuestras escalas permiten de forma específica medir las experiencias de compasión a nivel individual de forma bi-direccional, así como también, las prácticas organizacionales que podrían potenciar las conductas compasivas. Aunque el estudio de Lilius et al. (2008) en su versión cualitativa exploró el tipo y frecuencia de prácticas organizacionales compasivas, no se ha documentado una escala que mida las prácticas y políticas organizacionales compasivas. La medición de los aspectos organizacionales de la compasión es importante para así evidenciar el resultado que tienen las políticas y prácticas compasivas en el bienestar de los empleados.

Por otro lado, los resultados de esta investigación evidencian que más allá del sufrimiento observado en otros, las necesidades angustiantes pueden evocar las conductas compasivas. En esa dirección, el concepto de necesidades angustiantes permite tener una perspectiva más atemperada al contexto cultural hispano, para explicar y entender la compasión en el trabajo. Los ítems puestos a prueba en esta investigación operacionalizaron las necesidades angustiantes de las personas trabajadoras como antecedentes a las conductas compasivas.

A nivel de implicaciones prácticas, las tres escalas desarrolladas en esta investigación son útiles para futuras investigaciones, así como para realizar intervenciones de diagnóstico organizacional que integre el elemento de compasión laboral como parte de su análisis. Este tipo de evaluación permitiría tener una perspectiva más amplia, que resulte en ambientes de trabajo más humanizados, saludables y compasivos, y que redunden en mejorar la calidad de vida laboral y las metas organizacionales. Existe evidencia que sustenta que cuando los empleados se sienten respaldados por sus organizaciones, perciben que la organización se preocupa por su bienestar, lo que los motiva a mostrar apoyo a sus compañeros de trabajo y a la organización (Moon, et al., 2016; Ledoux et al., 2018).

Las tres escalas de compasión en el trabajo poseen excelentes propiedades psicométricas (estructura factorial, confiabilidad y varianza media extraída). De igual forma, la capacidad de discriminación de los ítems de las tres escalas adecuada. Las escalas presentaron buenos y aceptables índices de fiabilidad. A pesar de que las correlaciones entre las escalas y subescalas fluctuaron en magnitudes de moderada baja a moderada alta, cabe destacar que el análisis de validez discriminante confirma que cada instrumento mide un concepto diferente, sin que exista redundancia entre ellos. Estos resultados confirman que las escalas miden independientemente la compasión 
individual (de mí hacia otros y de otros hacia mí) y la compasión organizacional (políticas y prácticas organizacionales). Por tanto, esta investigación aporta tres perspectivas distintas al estudio y medición de la compasión laboral, desde un espectro más amplio cuando se compara con otras escalas existentes. En particular, es útil para realizar intervenciones de diagnóstico organizacional que incluya la medición de las dimensiones de la compasión individual y también organizacional en su análisis.

El uso de las tres escalas de compasión en el trabajo permitirá estudiar el fenómeno en otros contextos laborales hispanos, así como con otras variables organizacionales que aporten al nuevo conocimiento de la psicología ocupacional positiva. Las excelentes propiedades psicométricas de las escalas de compasión en el trabajo permitirán realizar inferencias válidas y confiables sobre el constructo en escenarios laborales de habla hispana.

\section{Fortalezas y Limitaciones}

Los resultados de nuestro estudio deben ser interpretados a la luz de sus limitaciones, pero sin que ello vaya en menoscabo de sus aportaciones. Una limitación del estudio es que la muestra original fue seleccionada por disponibilidad, por lo que no se pueden generalizar los resultados de los datos. Sin embargo, la muestra del estudio es una heterogénea en términos demográficos, lo que puede atenuar esta limitación. Nuestro estudio aporta una perspectiva más amplia del fenómeno de la compasión, tomando en consideración las necesidades angustiantes de las personas, distanciándonos de la noción del sufrimiento, de donde parten la mayoría de las investigaciones del fenómeno. Por otro lado, nuestro estudio aborda las tres operaciones psicológicas básicas (cognitiva, afectiva y conductual) de la compasión individual, así como las dimensiones de políticas y prácticas compasivas a nivel organizacional. Otra fortaleza de este estudio es que aborda el tema de la compasión laboral desde la psicología positiva en un contexto latinoamericano, hispano y caribeño.

\section{Uso de las Escalas de Compasión Laboral}

Las tres escalas de compasión en el trabajo son auto-administrables. La Escala Individual de Compasión en el Trabajo integra tres subescalas (cognición, afecto y conductas) para medir la compasión del individuo a otras personas. La Escala de Compasión de Compañeros de Trabajo mide las conductas compasivas de otros hacia el individuo. Mientras que la Escala de Compasión Organizacional mide las políticas y prácticas compasivas en la organización. Para calcular las puntuaciones de la Escala Individual de Compasión en el Trabajo es preciso hacerlo por subescala, donde se suman las puntuaciones de cada ítem de cada dimensión y se dividen entre 3. Para calcular las puntuaciones de la Escala de Compasión de Compañeros de Trabajo, se suman las puntuaciones de cada ítem y se divide entre 7. Para calcular las puntuaciones de la Escala de Compasión Organizacional es preciso hacerlo por subescala. Para la dimensión de Políticas Compasivas se suman las puntuaciones de cada ítem de cada dimensión y se dividen entre 3. Para la dimensión de Prácticas Compasivas se suman las puntuaciones de cada ítem de cada dimensión y se dividen entre 5 . Las puntuaciones de las tres escalas fluctuarán entre 0 (Nunca) hasta 4 (Siempre). En las tablas 2, 3 y 4 se describe la estructura de los ítems que componen la escala.

\section{Futuros Estudios}

El estudio hace una invitación a continuar investigando acerca de la compasión laboral desde la psicología positiva para aporta al bienestar y mejor funcionamiento humano y organizacional. Según plantea Salanova y Schaufeli (2000) la investigación desde este paradigma podría proveer respuestas a las organizaciones modernas en su proceso de adaptarse a los cambios desde una mirada positiva. Futuras investigaciones pudieran analizar cómo la compasión organizacional, predice la compasión individual y otros estados psicológicos de bienestar como el engagement en el trabajo y las emociones positivas. De igual forma, se recomienda estudiar la compasión laboral con otras variables como por ejemplo las conductas compasivas y el género, el conflicto trabajo-vida personal, las conductas de ciudadanía organizacional, justicia organizacional, la resiliencia en el trabajo y la responsabilidad social corporativa. Finalmente, es imperativo continuar ampliando la investigación del constructo en contextos multiculturales que ayuden en adelantar el conocimiento científico.

\section{CONCLUSIÓN}

La compasión es un fenómeno social que se manifiesta de forma natural en los escenarios de trabajo. 
Hoy día las organizaciones han despertado un interés en fomentar la compasión en el contexto laboral. La evidencia científica destaca los beneficios de potenciar la compasión a través de prácticas y políticas organizacionales dirigidas a crear ambientes de trabajo que sean más humanos, flexibles y que mejoren la calidad de vida laboral. Por ende, esta investigación llena un vacío en la medición de la compasión en Puerto Rico y otros contextos hispanos. El despertar de la compasión en el trabajo tiene un poder silencioso que "eleva a la gente y a las organizaciones" (portada del libro) (Worline \& Dutton, 2017). Este es un claro entendido de las atribuciones positivas que se dan a la compasión en el trabajo y el bienestar de las personas. En ese sentido, este estudio valida lo que nuestro amigo y colega el Dr. Noel Quintero, psicólogo clínico colombiano, nos resumió en una charla entre amigos, cuando le preguntamos: ¿Qué es para ti la compasión? Con el tono afable característico del Valle del Cauca nos dijo: "Amigos... la compasión no es otra cosa que el no ser indiferente a la necesidad ajena".

Financiamiento: La presente investigación no fue financiada por alguna entidad ni patrocinador.

Conflicto de Intereses: No existen conflictos de intereses de parte de los autores de la investigación.

Aprobación de la Junta Institucional para la Protección de Seres Humanos en la Investigación: Comité de Revisión Institucional (IRB) de la Universidad Carlos Albizu de San Juan, Puerto Rico (protocolo Fall-19-24).

Consentimiento o Asentimiento Informado: Todo participante completó un consentimiento informado.

Proceso de Revisión: Este estudio ha sido revisado por pares externos en modalidad de doble ciego.

\section{REFERENCIAS}

Avramchuk, A. S. \& Manning, M. R. (2014). Executive compassion: How executives construe compassion at work. Current Topics in Management, Center for Advanced Studies in Management, 17, 129-153.

Ato, M., \& López, J.J., \& Benavente, A. (2013). Un sistema de clasificación de los diseños de investigación en psicología. Anales de Psicología, 29(3),1038-1059.

https://www.redalyc.org/articulo.oa?id=16728244043

Barbot, J. \& Dodier, N. (2015). Dealing with compassion at work: strategic reflexivity among court lawyers. Sociologie du travail, 57, 43-62. https://doi.org/10.1016/j.soctra.2015.09.009

Blau, F. M. (1964). Exchange and power in social life. Wiley.
Bowlby, J. (1969). Attachment and loss: Vol. 1 Attachment. Basie Books.

Bowlby, J. (1973). Attachment and loss: Vol. 2. Separation: Anxiety and anger. Basic Books.

Brown, T.A. (2015). Confirmatory factor analysis for applied research ( $2^{\text {nd }}$ ed.). Guilford Press.

Cameron, K. (2017). Organizational compassion: manifestations through organizations. En S. L., Worline, M. C., Cameron, C. D., \& Doty, J. R. (Eds.), The Oxford Handbook of Compassion Science (421-431). Oxford University Press.

Cameron, K., Mora, C., Leutscher, T. \& Calarco, M. (2011). Effects of positive practices on organizational effectiveness. Journal of Applied Behavioral Science, 47(3), 266-308. https://doi.org/10.1177/0021886310395514

Choi, H.J., Lee, S., No, S.R. \& Kim, E.I. (2016). Effects of compassion on employees' self-regulation. Social Behavior and Personality, 44(7), 1173-1190.

https://doi.org/10.2224/sbp.2016.44.7.1173

Chu, L.C. (2014). Mediating positive moods: the impact of experiencing compassion at work. Journal of Nursing Management, 24, 59-69. https://doi.org/10.1111/jonm.12272

Davis, M. H. (1983). The effect of dispositional empathy on emotional reactions and helping: a multidimensional approach. Journal of Personality, 51, 167-184. https://doi.org/10.1111/j.1467-6494.1983.tb00860.x

Dutton, J.E., Workman, K.M., \& Hardin, A.E. (2014). Compassion at work. Annual Review of Organizational Psychology and Organizational Behavior, 1, 277-304. https://doi.org/10.1146/annurevorgpsych-031413-091221

Fornell, C., \& Larcker, D.F. (1981). Evaluating structural equation models with unobservable variables and measurement error. Journal of Marketing Reasearch, 18(2), 39-50. https://doi.org/10.2307/3151312

Gilbert, P. (2018). La mente compasiva: una nueva forma de enfrentarse a los desafíos vitales. Editorial Eleftheria.

Hernández-Sampieri, R., Fernández-Collado, C., \& BaptistaLucio, P. (2006). Analisis de los datos cuantitativos. Metodología de la investigación, 407-499.

Hur, W.M., Moon, T. \& Rhee, S.Y. (2016a). Exploring the relationship between compassion at work: the evaluative perspective of positive work-related identity, service employee creativity, and job performance. Journal of Service Markeing, 30(1), 103114. https://doi.org/10.1108/JSM-05-2014-0180

Hur, W.M., Moon, T.W. \& Ko, S.H. (2016b). How employees' perceptions of CSR increase employee creativity: mediating mechanisms of compassion at work and intrinsic motivation. Springer Science+Business Media Dordrecht. https://doi.org/10.1007/s10551-016-3321-5

Kline, T. J. (2005). Psychological testing: a practical approach to design and evaluation. Sage Publications.

Ledoux, K., Forchuk, C, Higgins, C. \& Rudnick, A. (2018). The effect of organizational and personal variables on the ability to 
practice compassionately. Journal of Applied Nursing Research, 41, 15-20. https://doi.org/10.1016/j.apnr.2018.03.001

Mertens, D. (2004). Research and Evaluation in Education and Psychology: Integrating Diversity with Quantitative, Qualitative, and Mixed Methods.

Montero, I., \& León, O. G. (2007). A guide for naming research studies in psychology. International Journal of Clinical and Health Psychology, 7(3), 847-862.

Lilius, J.M., Worline, M.C., Maitlis, S., Kanov, J. Dutton, J.E., \& Frost, P. (2008). The contours and consequences of compassion at work. Journal of Organizational Behavior, 29, 193-218. https://doi.org/10.1002/job.508

Lupoli, M.J., Jampol, L. \& Oveis, C. (2017). Lying because we care: compassion increases prosocial lying. Journal of Experimental Psychology: General, 146(7), 1026-1042. https://doi.org/10.1037/xge0000315

Madden, L.T., Duchon, D. \& Plowman, D.A. (2012). Emergent organizational capacity for compassion. Academy of Management Review, 37(4), 689-708. http://dx.doi.org/!0.5465/amr.2010.0424

Martínez Lugo, M., Méndez Collada, V., \& Maeso Flores, R. (1999). La centralidad de trabajo y los intereses de vida en una muestra de empleados jóvenes y envejecidos en Puerto Rico. Revista Puertorriqueña de Psicología, 12, 77-91.

Martínez, M. E., Morales, O., Rodríguez, O., Rosa, W., \& Sánchez, S. (2016). Civilidad, incivilidad y el bienestar asociado al trabajo en un grupo de personas empleadas en Puerto Rico. Revista Interamericana de Psicología Ocupacional, 28(1), 45-55.

Moon, T.W., Hur, W.M., Ko, S.H., Kim, J.W., \& Yoon, S.W. (2014). Bridging corporate social responsibility and compassion at work: relations to organizational justice and affective organizational commitment. Career Development International, 19(1), 49-72.

Moon, T.W., Hur, W. M., Ko, S.H., Kim, J.W. \& Yoo, D.K. (2016). Positive work-related identity as a mediator of the relationship between compassion at work and employee outcomes. Human Factors and Ergonomics in Manufacturing $\mathcal{E}$ Service Industries, 26(1) 84-94. https://doi.org/10.1108/CDI-05-2013-0060

Pommier, E. A. (2011). The Compassion Scale. Dissertation Abstracts International Section A: Humanities and Social Science, 72, 1174 .

Ray, S.L., Wong, C., White, D. \& Heaslip, K. (2013). Compassion satisfaction, compassion fatigue, work life conditions, and burnout among frontline mental health care professionals. Traumatology, 19(4), 255-267. https://doi.org/10.1177/1534765612471144

Reich, W. T. (1989). Speaking of suffering: A moral account of compassion. Soundings, 72, 83-108. http://www.jstor.org/stable/41178467
Rhee, S.Y., Hur, W.M. \& Kim, M. (2017). The relationship of coworker incivility to job performance and the moderating role of self-efficacy and compassion at work: the Job Demands-Resources (JD-R) Approach. Journal of Bussiness Psychology, 32, 711-726. https://doi.org/10.1007/s10869-0169469-2

Rodríguez-Montalbán, R. (2018). La compasión en el entorno laboral: concepto y medida. Protocolo IRB núm. Fall 1822. Universidad Carlos Albizu, San Juan, PR.

Rosario-Hernández, E., Millán, L. V. R., Rivera, L., Rivera, G., Padovani, C., Plaza, Y., \& Gallardo, R. (2016). La incivilidad en el lugar de trabajo y su relación con el bienestar psicológico en una muestra de empleados en Puerto Rico. Revista Interamericana de Psicología Ocupacional, 29(1), 5-19.

Rosario-Hernández, E., Millán, L. V. R., Rodríguez Irizarry, A., Velázquez Lugo, Á. V., Rosario Nieves, I. R., \& Zapata Solá, A. Z. (2013). La relación entre la manifestación de conductas laborales contraproducentes, estresores laborales, emociones y acoso psicológico: El efecto moderador de las emociones negativas. Revista Interamericana de Psicología Ocupacional, 32(1), $31-52$.

Salanova, M. \& Schaufeli, W.B. (2000). Exposure to information technologies and its relation to burnout. Behavior $\mathcal{E}$ Information Technology, 19, 385-392. https://doi.org/10.1080/014492900750000081

Satorra, A. \& Bentler, P. (2001). A scaled difference chi-square test statistic for moment structure analysis. Psychometrika, 66(4), 507-514. https://doi.org/10.1007/BF02296192

Stamm, B. H. (2010). The concise ProQOL manual (2nd ed.), Pocatello, ID: ProQOL.org.

Velasco, M., Navarro, J. \& Rueff-Lopes, R. (2015). Teoría de los acontecimientos afectivos: Revisión de su impacto y desarrollos en el estudio del afecto en las organizaciones. Revista Psicología: Organizações e Trabalho. 17, 30-38. http://dx.doi.org/10.17652/rpot/2017.1.12106

Wagaman, M. A., Geiger, J.M., Schockley, C. \& Segal, E.A. (2015). The role of empathy in burnout, compassion satisfaction, and secondary traumatic stress among social workers. National Association of Social Workers, 201-209. https://doi.org/10.1093/sw/swv014

Weiss, H. M., \& Cropanzano, R. (1996). Affective events theory: A theoretical discussion of the structure, causes and consequences of affective experiences at work. In B. M. Staw \& L. L. Cummings (Eds.), Research in organizational behavior: An annual series of analytical essays and critical reviews, (Vol. 18, pp. 1-74). Greenwich: Elsevier Science/JAI Press.

Worline, M. C. \& Dutton, J. E. (2017). Awakening compassion at work: the quiet power that elevates people and organizations. Berrett-Koehler Publishers. 